УДК 553.78

\title{
ПРИМЕНЕНИЕ КОМПЬЮТЕРНОГО МОДЕЛИРОВАНИЯ ДЛЯ УСТОЙЧИВОЙ ЭКСПЛУАТАЦИИ ТЕРМАЛЬНЫХ ПОДЗЕМНЫХ ВОД ХАНКАЛЬСКОГО МЕСТОРОЖДЕНИЯ
}

\section{Фархутдинов Анвар Мансурович',} anvarfarh@mail.ru

\author{
Фархутдинов Исхак Мансурович', \\ iskhakgeo@gmail.com
}

\author{
Черкасов Сергей Владимирович², \\ sergy@sgm.ru \\ Исмагилов Рустем Айратовичз, , \\ rustem_ismagilov@bk.ru

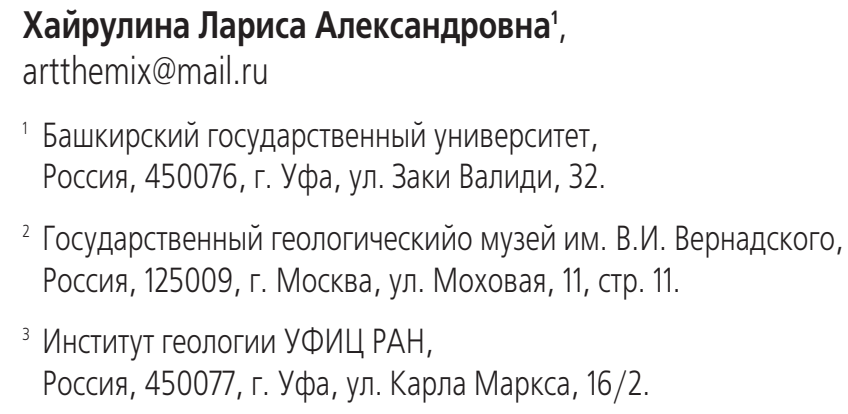

Актуальность исследования обусловлена необходимостью достижения устойчивости в эксплуатации термальных подземных вод. Снижение температуры геотермального резервуара при обратной закачке использованного флюида является важнейшей проблемой долгосрочной эксплуатации, прогноз которой возможен с помощью компьютерного моделирования.

Цель: дать прогноз изменения температурного режима в ходе эксплуатации Ханкальского месторождения, рассмотреть различные сценарии разработки и предложить возможные решения достижения устойчивости в разработке месторождения.

объекты: Ханкальское месторождение; дублетная циркуляционная система Ханкальской геотермальной станции.

Методы: компьютерное моделирование изменения температуры ресурса термальных подземных вод; оценка и анализ возможных вариантов периодической эксплуатации месторождения; оценка суммарной выработки тепла и конечной темпертуры геотермального резервуара на момент остановки для различных вариантов эксплуатации.

Результаты. На Ханкальском месторождении снижение температуры в продуктивной скважине начнется через 6-7 лет после разработки в запланированном режиме. Периодическая короткая эксплуатация и остановка (10-летний цикл, 100 лет суммарно) позволяют выработать больше тепла по сравнению с более продолжительной эксплуатацией (50-летний цикл, 100 лет суммарно), вместе с тем второй вариант дает возможность достичь более высокого восстановления температуры в продуктивной скважине. В случае значительного снижения температуры после некоторого периода эксплуатации XIII пласта предлагается периодическое использование различных продуктивных горизонтов (IV-VII, XVI или XXII) для обеспечения устойчивости работы геотермальной станции. Разработка ХІІІ пласта возможна после периода остановки благодаря относительно высокой скорости восстановления температуры. Следует размещать скважины параллельно двум главным разломам Ханкальского месторождения, при этом продуктивную скважину - в южной, а нагнетательную - в северной части, так как естественный поток подземных вод может замедлить расширение холодного фронта до продуктивной скважины. Установка и периодическое использование двух или более циркуляционных систем могут обеспечить наибольшую устойчивость при долгосрочной эксплуатации. Однако рациональность этого метода, требующего больших дополнительных затрат на начальном этапе реализации проекта, представляет собой сложный вопрос. Выбор режима эксплуатации требует учета гидрогеологических, геотермальных, экономических и технических аспектов при совместном участии гидрогеологов, специалистов по геотермии, технологов и экономистов.

\section{Ключевые слова:}

Возобновляемые ресурсы энергии, термальные подземные воды, устойчивая эксплуатация, дублет, моделирование, Ханкальское месторождение.

\section{Введение}

Значительное внимание в последние годы уделяется возобновляемым источникам энергии, использование которых считается одним из показателей развития страны. Важное значение среди них имеют термальные подземные воды. Несмотря на то, что в России использование термальных вод экономически уступает эксплуатации газовых и угольных станций [1], экологическая чистота и возобновляемость делает их применение перспективным [2]. Возобновляемость характеризуется тем, что «энергия, извлекаемая из возобновляемого источника, всегда естественным образом восполняется дополнительным количеством энергии, и замена происходит в аналогичном масштабе времени, что и извлечение» [3]. В то же время ресурс тер- 
мальных подземных вод может быть исчерпан в результате чрезмерной эксплуатации. Залогом достижения устойчивости в эксплуатации является мониторинг [4] и рациональный режим разработки, который подразумевает поддержание баланса в извлечении и восстановлении ресурса. Для симуляции поведения сложной геотермальной системы при различных условиях разработки используется математическое моделирование, что позволяет решать проблему устойчивости эксплуатации [5-12].

Компьютерное моделирование в этой отрасли началось с 1970-х гг., когда создание крупных гидротехнических сооружений и эксплуатация месторождений подземных вод определили необходимость гидрогеологических прогнозов и развития теории динамики подземных вод [13]. Разработано множество программ для моделирования тепломассопереноса в системах водоносных горизонтов (FEFLOW [14], MODFLOW [15], Metis [16], Comsol, Tough2 [17], Marthe [18], Opengeosys [19] и др.). Они дают возможность спрогнозировать и выбрать правильный режим эксплуатации геотермальных ресурсов, в частности, для учета эффекта обратной закачки.
В настоящей статье математическое моделирование применяется для прогноза изменения температурного режима и разработки рекомендаций по эксплуатации термальных вод Ханкальского месторождения.

\section{Материалы и методы}

Ханкальское месторождение термальных подземных вод

Месторождение расположено в Чеченской Республике, в $10 \mathrm{kм} \mathrm{к} \mathrm{юго-востоку} \mathrm{от} \mathrm{г.} \mathrm{Грозного}$ (рис. 1).

Территория относится к Восточно-Предкавказскому артезианскому бассейну площадью около 250 тыс. км$^{2}$ [20]. Термальные воды содержатся в многопластовой системе караган-чокракских отложений среднего миоцена, где выделяются до 22 продуктивных пластов, разделенных глинистыми прослойками. Зона питания расположена на юге Чеченской Республики, после инфильтрации воды движутся в северном направлении (рис. 2).

Факторами формирования геотермальных ресурсов данной территории являются высокие значения теплового потока, движение подземных вод и структурно-тектонические особенности (нагрев

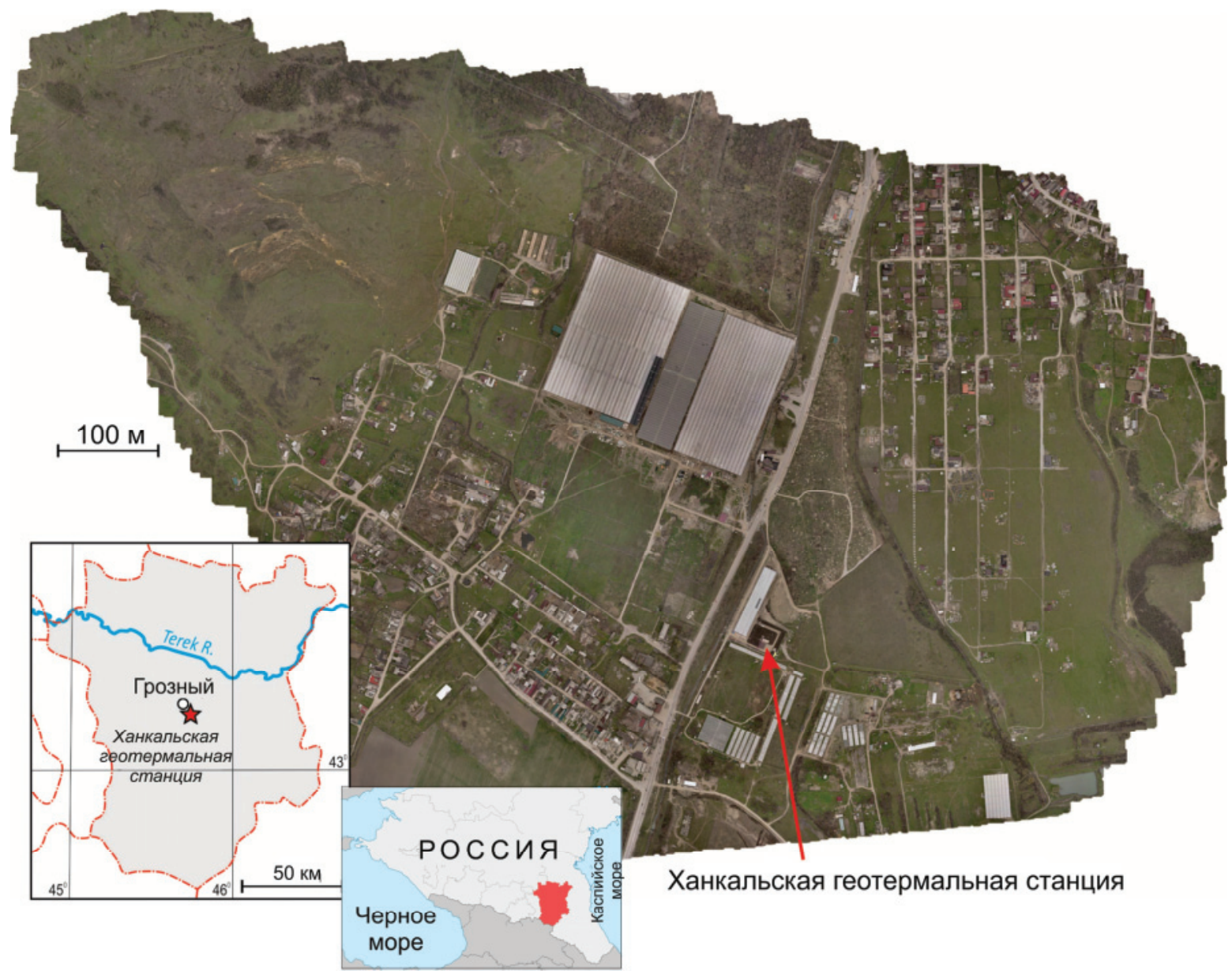

Рис. 1. Расположение Ханкальского месторождения

Fig. 1. Location of the Khankala field 


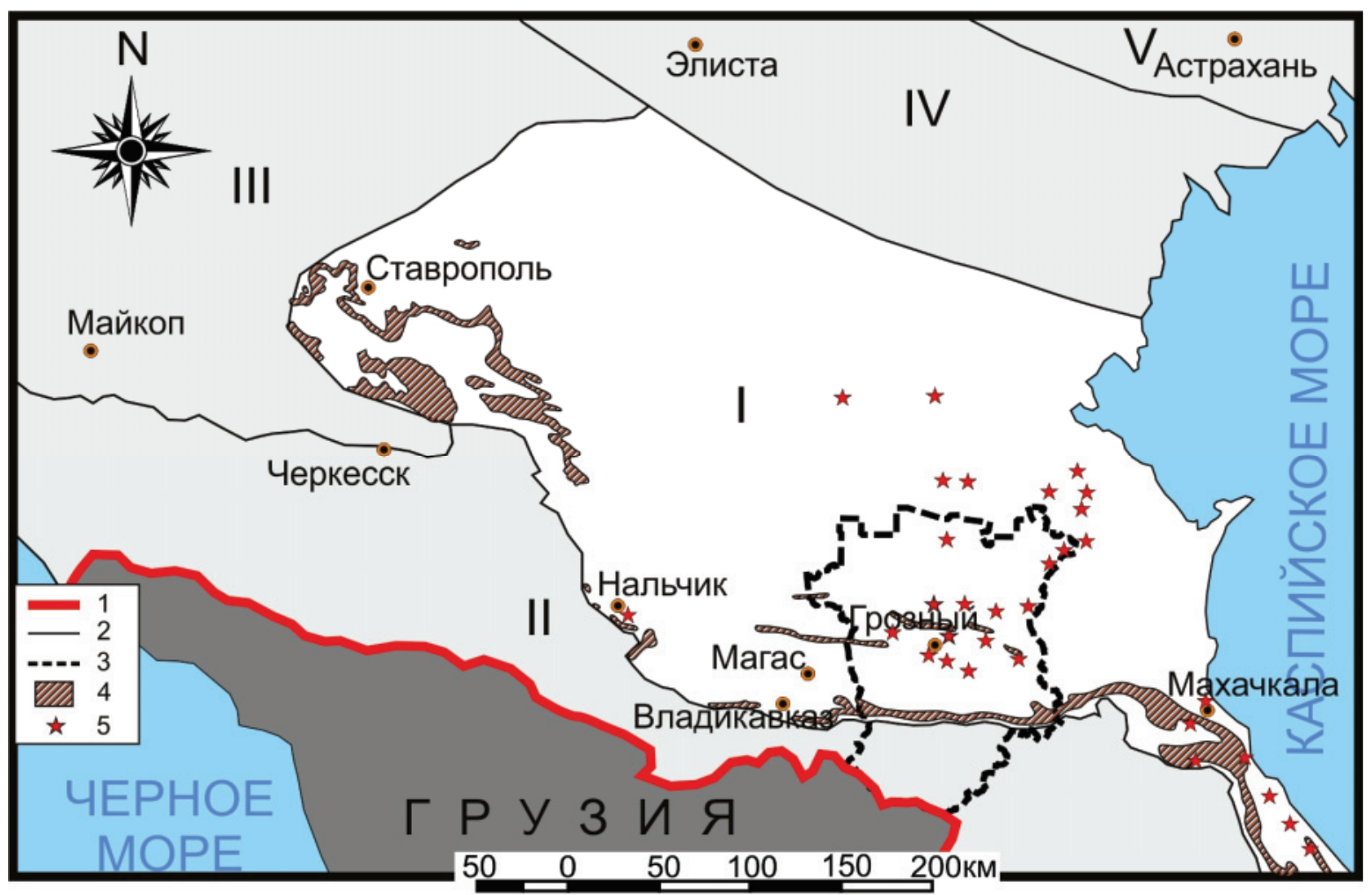

Pис. 2. Восточно-Предкавказский артезианский бассейн - схематическая карта по И.Ю. Дежниковой с измененияли [2015] [21]. Условные обозначения: 1 - госграница, 2 - границы гидрогеологических структур, 3 - гранииа Чеченской Республики, 4 - выходы караган-чокракских отложений на поверхность, 5 - месторождения терлальных подзелных вод; I-V артезианские бассейны: I - Восточно-Предкавказский, II - Кавказская гидрогеологическая складчатая область, III - Азово-Кубанский, IV - Ергенинский, $V-\Pi$ - икаспийский

Fig. 2. East Ciscaucasian Artesian Basin - schematic map after I.U. Dezhnikova with modifications [2015] [21]. Legend: 1 is the state border, 2 is hydrogeological structures border, 3 is the border of the Chechen Republic, 4 are the Karagan-Chokrak deposits outcrops, 5 are the geothermal fields; I-V artesian basins: I - the East Ciscaucasian, II - the Caucasian hydrogeological folded region, III - the Azov-Kuban, $I V$ - the Ergeninsky, $V$ - the Caspian

воды в синклинальных прогибах, а затем ее подъем на поверхность), а также литология горных пород - продуктивные пласты заключены между глинами сармата и майкопа, которые способствуют сохранению тепла [22].

Территория месторождения ограничена двумя разломами (рис. 3).

В начале 2016 г. на базе наиболее перспективного XIII продуктивного пласта была запущена геотермальная станция. Мощность объекта составляет 22,8 ГДж/час с парниковым комплексом в качестве потребителя [23]. Эксплуатация проводится с применением «дублета» (рис. 4), состоящего из продуктивной и нагнетательной скважин с полной обратной закачкой использованного флюида. Температура получаемой воды составляет $95{ }^{\circ} \mathrm{C}$, обратной закачки равна $45{ }^{\circ} \mathrm{C}$ [24].

Обратная закачка использованной жидкости позволяет минимизировать возможное отрицательное воздействие на окружающую среду, а также поддерживать пластовое давление и, следовательно, высокие дебиты. Однако у данного метода есть главный недостаток - снижение температуры в водоносном горизонте, для прогноза которого применяется компьютерное моделирование.

\section{Компьютерное моделирование}

Компьютерная программа Metis [16] моделирует поток подземных вод и тепломассоперенос в пористых или трещинных водоносных горизонтах. Перед моделированием определяется геометрия, параметры системы, а также начальные и граничные условия с использованием распределения температуры и структурной карты XIII пласта, полученных после геостатистической оценки [25]. Кроме того, привлекались фондовые данные, в том числе результаты испытаний скважин, некоторые теплофизические параметры, а также средние значения теплопроводности и теплоемкости различных типов пород из литературных источников [26] (табл. 1).

Граничные условия (рис. 5):

- нагнетание воды $200 \mathrm{~m}^{3} /$ ч 7 месяцев в году (отопительный период) с постоянным потоком тепла 10051 Мватт (отвечает температуре обратной закачки $\left.45^{\circ} \mathrm{C}\right)$;

- тепловой поток от основания модели равный $82 \mathrm{мB}$ т/ м $^{2}$ (по М.К. Курбанову [20]).

- постоянный напор на границах сетки для создания регионального потока подземных вод либо непроницаемые границы на основных двух разломах. 


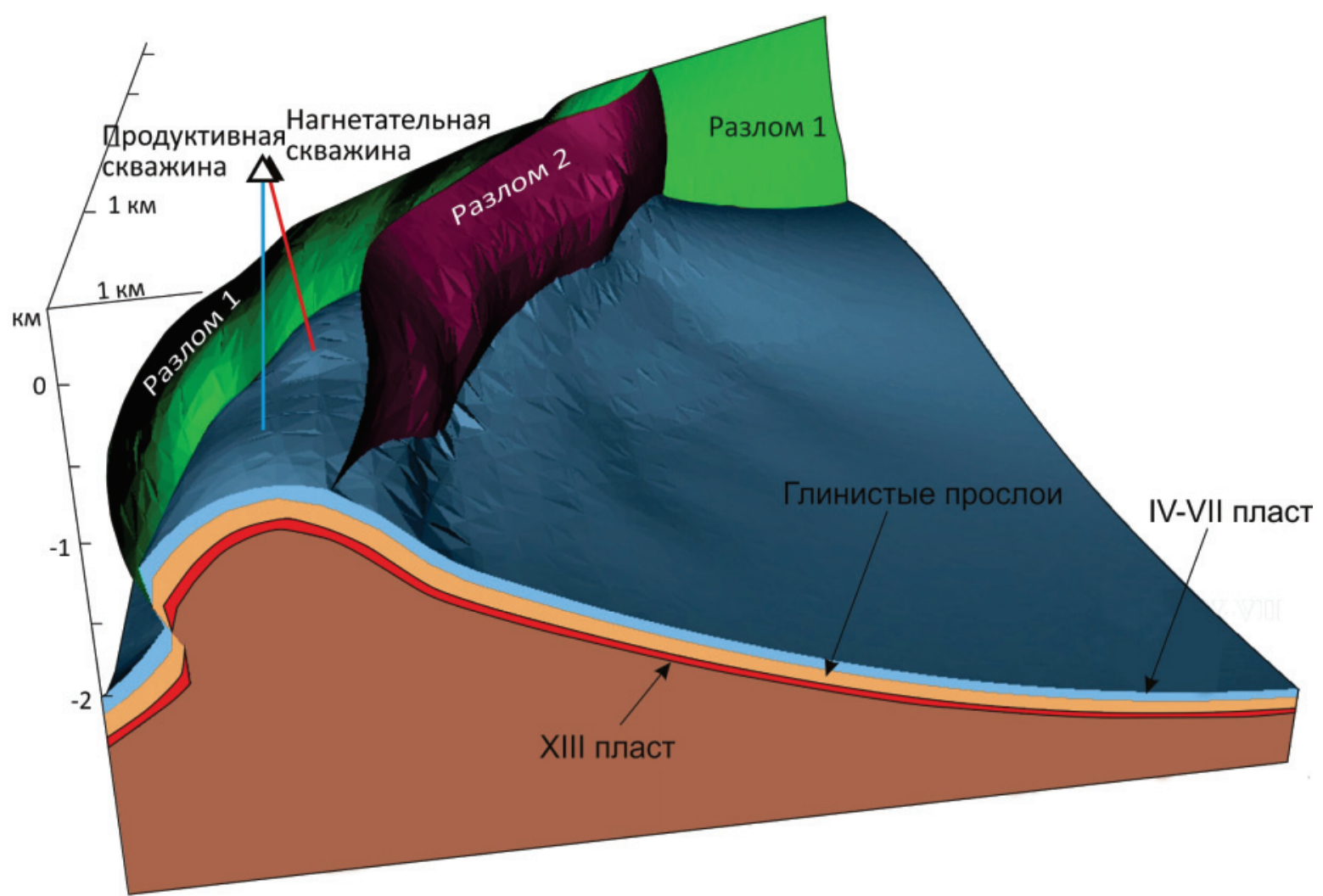

Рис. 3. Трехмерная геологическая модель Ханкальского месторождения

Fig. 3. Khankala field $3 D$ geological model

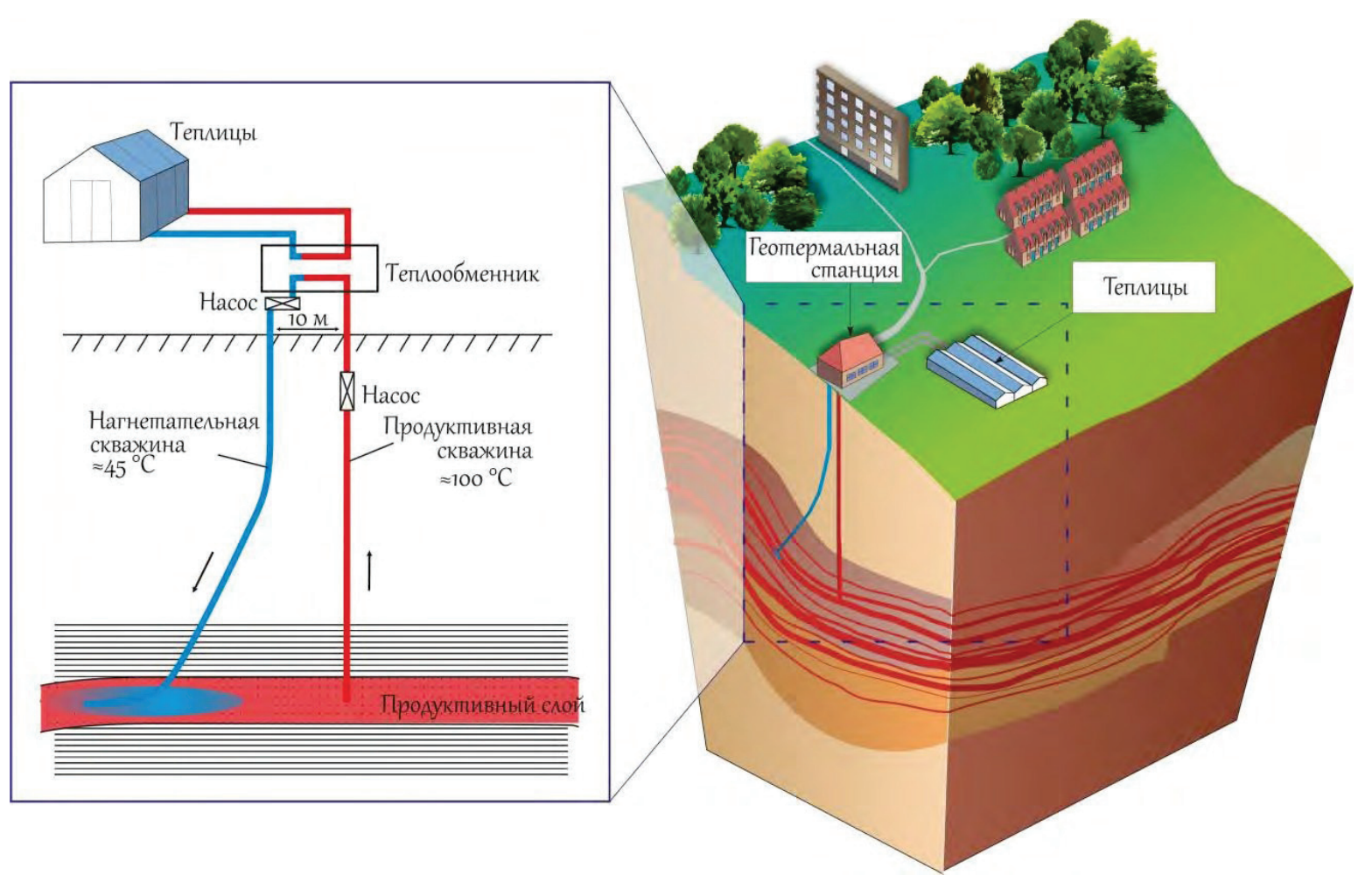

Рис. 4. «Дублетная» ииркуляиионная система Ханкальского месторождения

Fig. 4. Khankala field «doublet» circulation system 
таблица 1. Начальные параметры моделирования

Table 1. Modelling initial conditions

\begin{tabular}{|c|c|c|c|c|c|c|c|c|c|}
\hline \multicolumn{2}{|c|}{$\begin{array}{c}\text { Глубина залегания, м } \\
\text { Depth, m }\end{array}$} & \multirow{2}{*}{\begin{tabular}{|c|} 
Мощость, \\
м \\
Thick- \\
ness, m \\
\end{tabular}} & \multirow{2}{*}{$\begin{array}{c}\text { Геологический разрез } \\
\text { Geological section }\end{array}$} & \multirow{2}{*}{\multicolumn{2}{|c|}{\begin{tabular}{|c|} 
Теплопровод- \\
ность, Вт $/\left(\mathrm{s}^{\circ}{ }^{\circ} \mathrm{C}\right)$ \\
Thermal conduc- \\
tivity, $\mathrm{W} /\left(\mathrm{m} \cdot{ }^{\circ} \mathrm{C}\right)$
\end{tabular}}} & \multirow{2}{*}{\multicolumn{2}{|c|}{\begin{tabular}{|c|} 
Объемная теплоем- \\
кость, МДж $/ \mathrm{m}^{3} /{ }^{\circ} \mathrm{C}$ \\
Volumetric heat \\
capacity, $\mathrm{MJ} / \mathrm{m}^{3} /{ }^{\circ} \mathrm{C}$
\end{tabular}}} & \multirow{2}{*}{\begin{tabular}{|c|} 
Проница- \\
емость, ${ }^{2}$ \\
Permeabi- \\
lity, ${ }^{2}$ \\
\end{tabular}} & \multirow{2}{*}{\begin{tabular}{|c|} 
Коэффициент \\
упругой \\
емкости, м $^{-1}$ \\
Storativity, $\mathrm{m}^{-1}$
\end{tabular}} \\
\hline \begin{tabular}{|l} 
от (верх) \\
from (top)
\end{tabular} & $\begin{array}{c}\text { до (низ) } \\
\text { to (bottom) }\end{array}$ & & & & & & & & \\
\hline 0 & 35 & 35 & $\begin{array}{l}\text { Четвертичные отложения } \\
\text { Quaternery sediments }\end{array}$ & 2,1 & \multirow{3}{*}{1,53} & 2,1 & \multirow{3}{*}{2,23} & \multirow{3}{*}{ - } & \multirow{3}{*}{ - } \\
\hline 35 & 700 & 665 & $\begin{array}{l}\text { Сарматские отложения } \\
\text { Sarmatian sediments }\end{array}$ & 1,4 & & 2,2 & & & \\
\hline 700 & 843 & 143 & $\begin{array}{l}\text { Караганские отложения (до XIII пласта) } \\
\text { Karagan sediments (above the XIII layer) }\end{array}$ & 2 & & 2,4 & & & \\
\hline 843 & 890 & 47 & $\begin{array}{l}\text { XIII продуктивный пласт } \\
\text { XIII productive layer }\end{array}$ & 2,3 & 2,3 & 2,486 & 2,486 & $6.77 \mathrm{e}-13$ & $5 e-6$ \\
\hline 890 & 1290 & 400 & $\begin{array}{l}\text { Караган-чокракские отложения } \\
\text { (после XIII пласта) } \\
\text { Karagan-Chokrak sediments } \\
\text { (below the XIII layer) }\end{array}$ & 1,8 & \multirow[t]{2}{*}{1,5} & 2,4 & \multirow[t]{2}{*}{2,28} & \multirow[t]{2}{*}{-} & \multirow[t]{2}{*}{-} \\
\hline 1290 & 2500 & 1210 & $\begin{array}{l}\text { Майкопские отложения } \\
\text { Maikop sediments }\end{array}$ & 1,4 & & 2,25 & & & \\
\hline
\end{tabular}

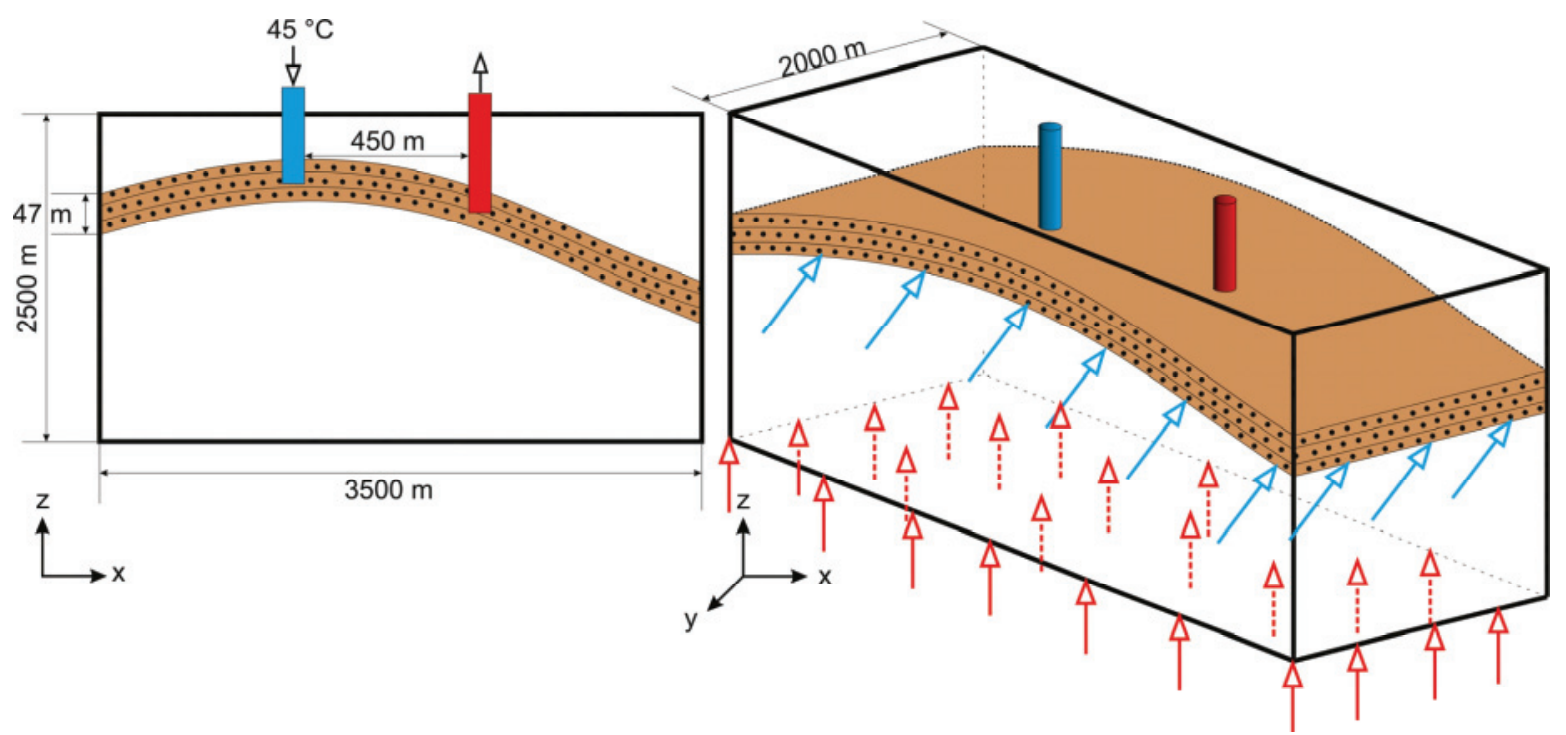

Pис.5. Схематическое изображение модели дублета. Красные и синие стрелки указывают направление теплового потока и естественного движения подземных вод, соответственно

Fig. 5. Schematic representation of the doublet model. Red and blue arrows indicate the direction of heat flow and natural groundwater movement, respectively

Процессы переноса жидкости и тепла связаны: на каждом этапе программа производит поочередное решение уравнений методом конечных элементов.

\section{Результаты}

Для оценки возможного влияния двух главных разломов Ханкальского месторождения рассматривались две различные гипотезы, одна - с учетом естественного регионального движения подземных вод, другая - с учетом их непроницаемости.

Результаты моделирования сравнивались с аналитическим решением изменения температуры "дублета» [27]. Для аналитического решения был установлен усредненный постоянный дебит равный $117 \mathrm{~m}^{3} /$ ч (рис. 6, табл. 2). таблица 2. Результаты моделирования

Table 2. Modeling results

\begin{tabular}{|l|c|c|}
\hline \multicolumn{1}{|c|}{ Условие } & $\begin{array}{c}\Delta T=1{ }^{\circ} \mathrm{C}, \text { лет } \\
\Delta T=1{ }^{\circ} \mathrm{C}, \text { years }\end{array}$ & $\begin{array}{c}\Delta T,{ }^{\circ} \mathrm{C}(50 \text { лет) } \\
\Delta T,{ }^{\circ} \mathrm{C}(50 \text { years })\end{array}$ \\
\hline $\begin{array}{l}\text { Непроницаемые разломы } \\
\text { Impermeable faults }\end{array}$ & 7,56 & $-20,74$ \\
\hline $\begin{array}{l}\text { Eстественный поток подземных вод } \\
\text { Natural groundwater flow }\end{array}$ & 10,1 & -12 \\
\hline $\begin{array}{l}\text { Aналитическое peшение } \\
\text { Analytical solution }\end{array}$ & 6,3 & $-24,07$ \\
\hline
\end{tabular}

«Тепловой прорыв» происходит раньше, и температура уменьшается быстрее в случае аналитического решения (аналогичные результаты были получены с помощью 2D моделирования в программе Metis с однородным распределением температуры 


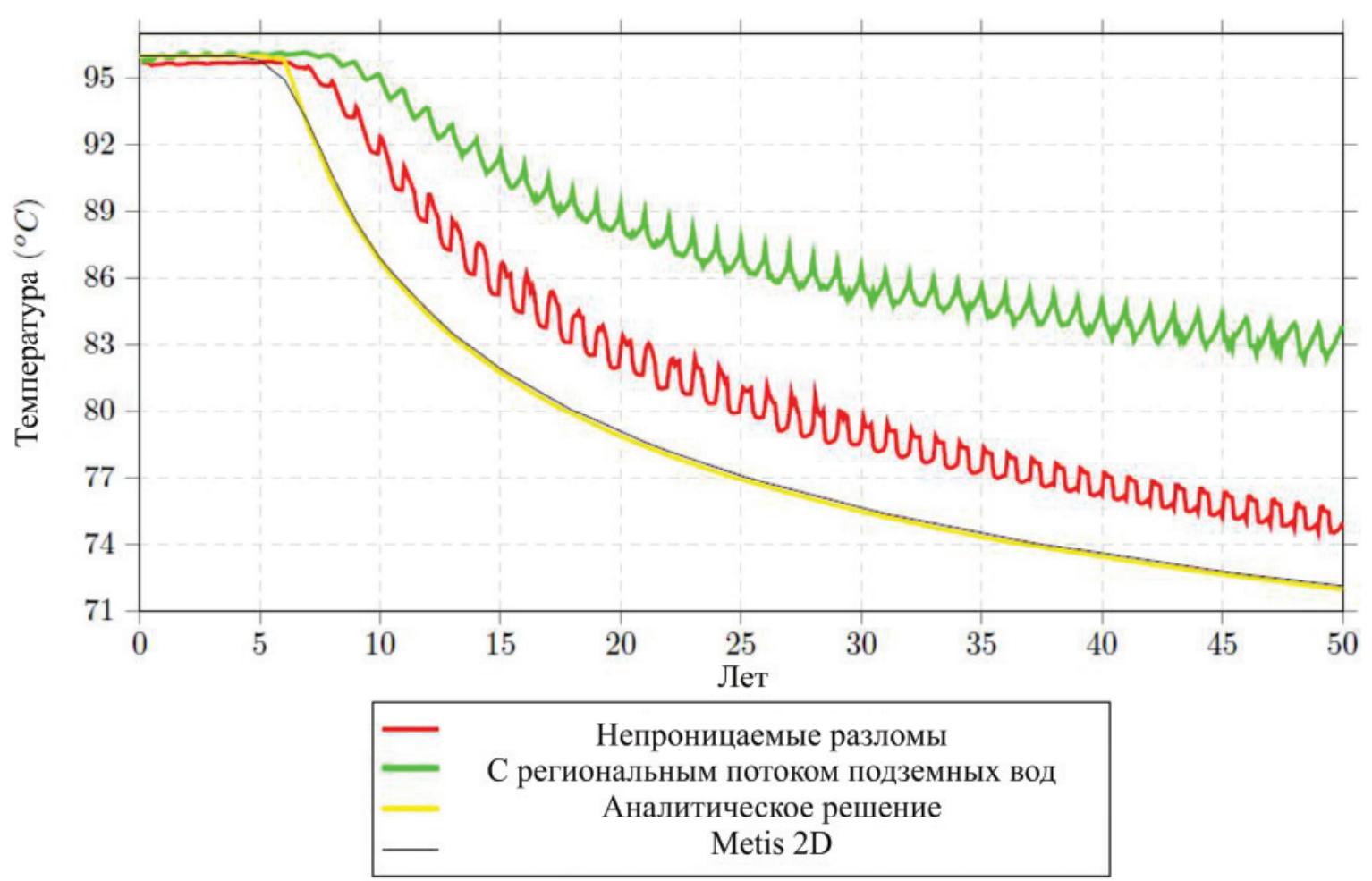

Рис. 6. Результаты моделирования: изленение телпературы на эксплуатационной скважине

Fig. 6. Modeling results: production well temperature change

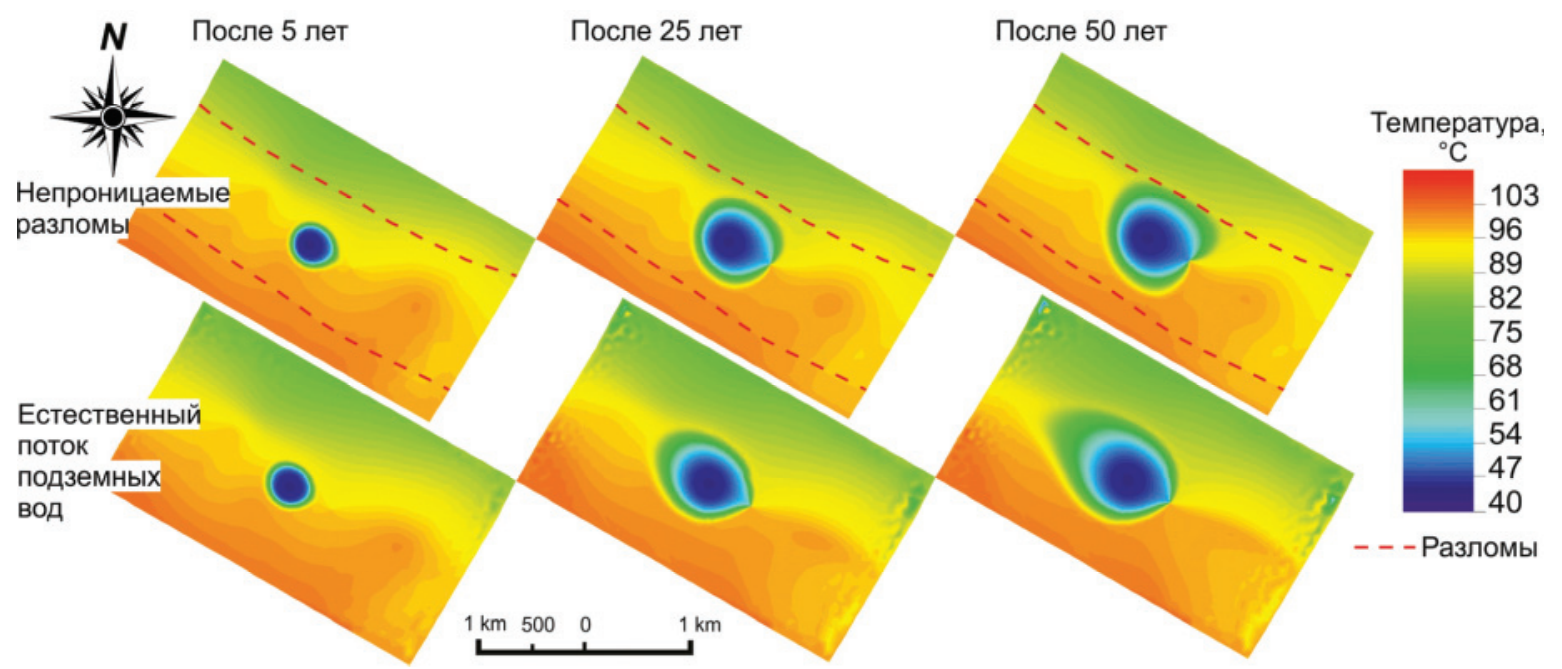

Рис. 7. Результаты моделирования: изменение температуры в ХІІІ пласте

Fig. 7. Modeling results: the XIII layer temperature change

(рис. 6)), так как температура продуктивного пласта и окружающих пород одинакова. В случае же 3D моделирования температура получена после геостатистической оценки и распределена неравномерно с более высокими значениями глубже и к югу от эксплуатационной скважины (рис. 7).

Дальнейшее исследование посвящено моделированию восстановления ресурса продуктивного пласта. Рассматривались различные возможные сценарии эксплуатации: постоянная эксплуатация и чередование разработки и остановки с циклами в 10, 25 и 50 лет (100 лет суммарно) (рис. 8).

Общее количество возможной выработки тепла рассчитывалось по формуле [28], адаптированной для установки «дублетных» циркуляционных систем (табл. 3):

$$
G=10^{-3} Q \eta C\left(t_{\text {добычи }}-t_{\text {закачки }}\right),
$$

где $G$ - тепловые ресурсы, ГДж/сутки; $Q$ - дебит, $\mathrm{M}^{3} /$ сутки; $t_{\text {добычи }}$ - температура добываемой воды, ${ }^{\circ} \mathrm{C}$; 


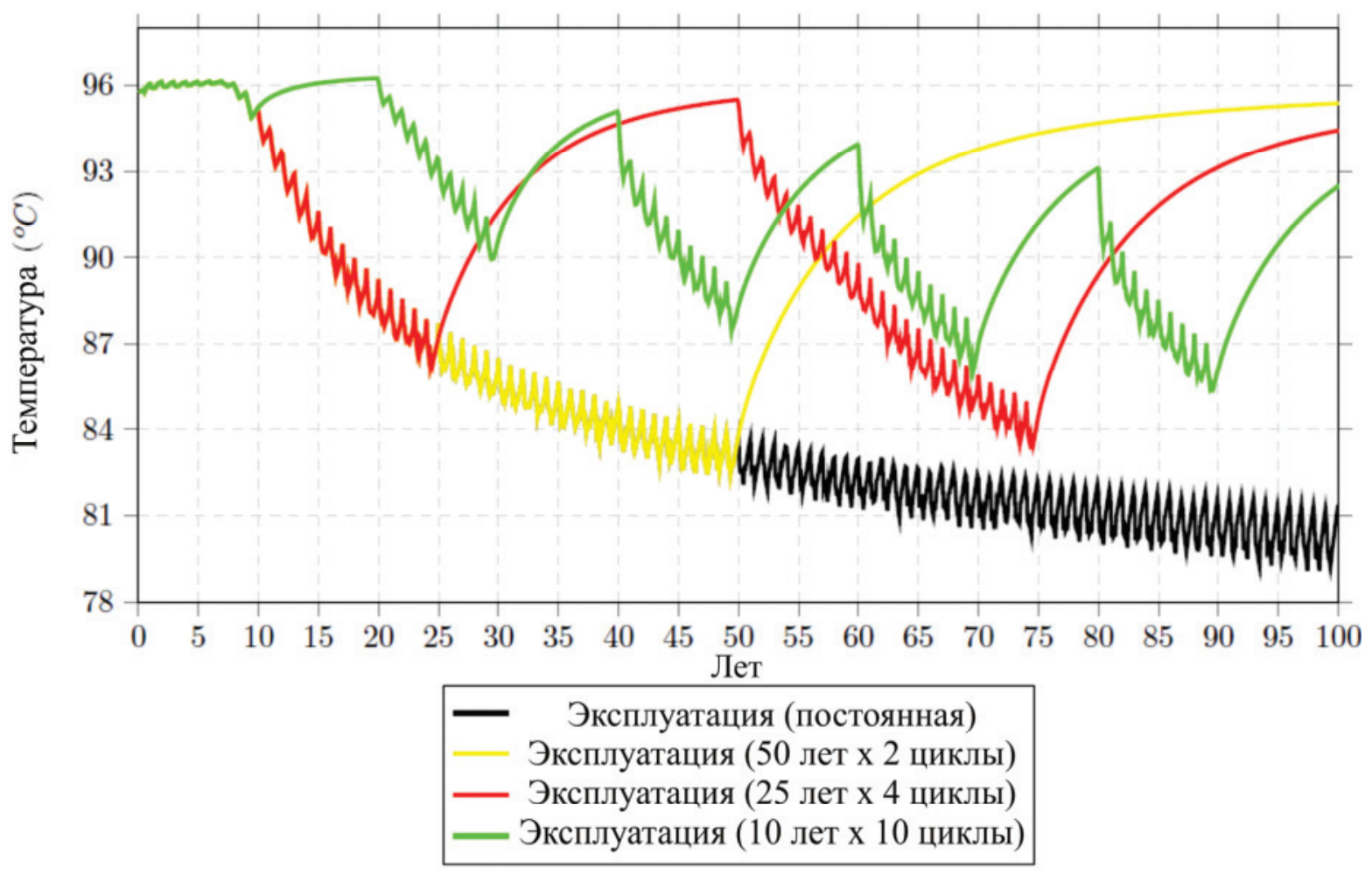

Рис. 8. Результаты моделирования: изленение телпературы в эксплуатационной скважине для различных сиенариев эксплуатации

Fig. 8. Modeling results: production well temperature change in case of different exploitation scenarios

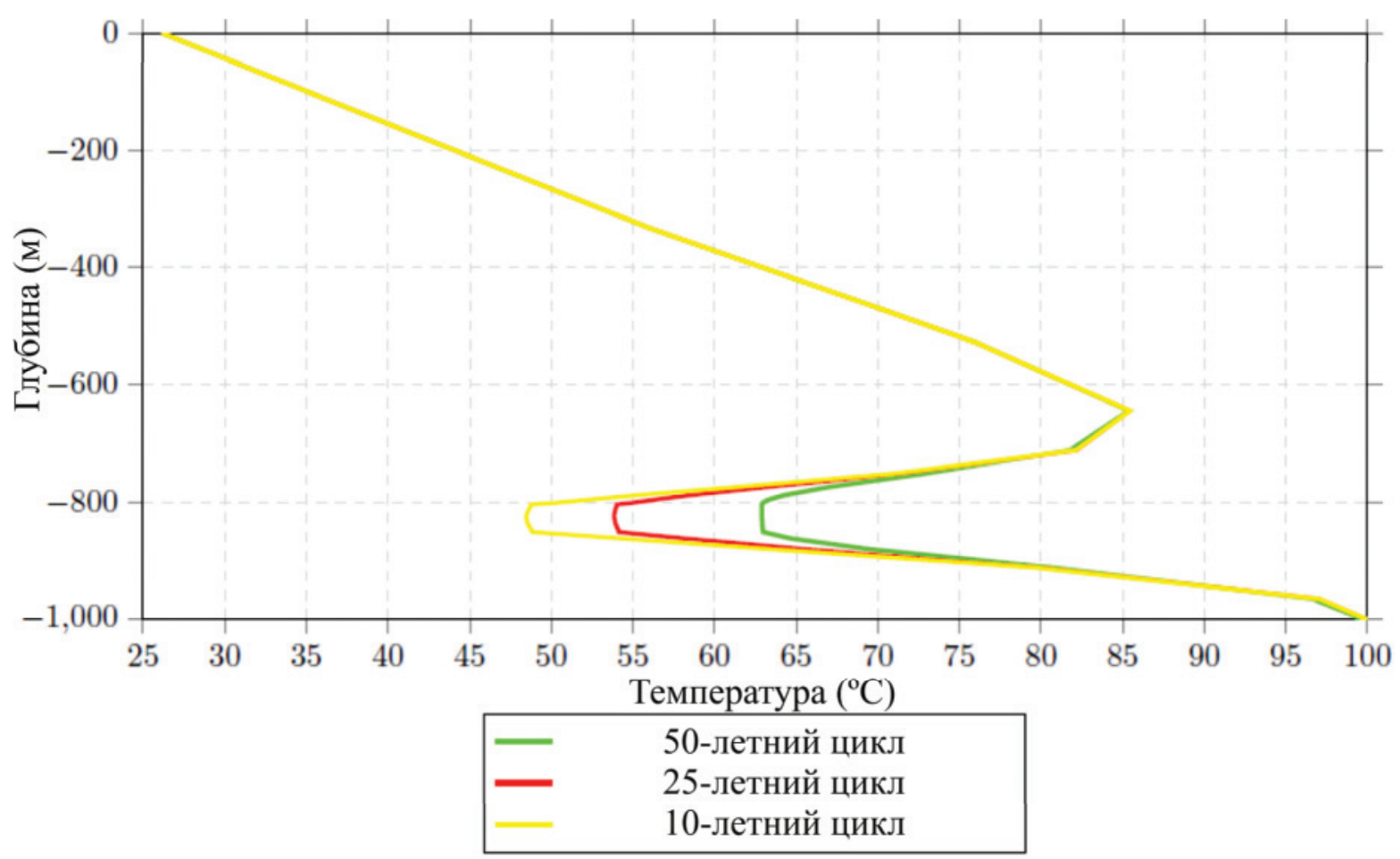

Рис. 9. Температурный профиль на продуктивной скважине после 100 лет эксплуатации

Fig. 9. Temperature section at the production well after 100 years of exploitation 
$t_{\text {закачки }}$ - температура закачиваемой воды, $45{ }^{\circ} \mathrm{C} ; \mathrm{C}-$ удельная теплоемкость воды $\left(4,186\right.$ кДж/кг $\left.{ }^{\circ} \mathrm{C}\right)$; $\eta$ - эффективность теплообменника 0,9 .

Таблица 3. Различные варианты эксплуатащии ХІІІ пласта Table 3. XIII layer different exploitation scenarios

\begin{tabular}{|c|c|c|c|c|c|}
\hline 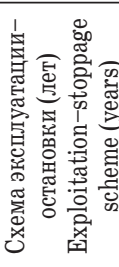 & 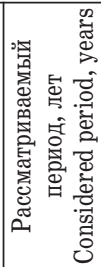 & 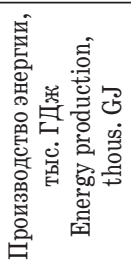 & 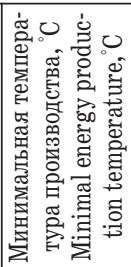 & 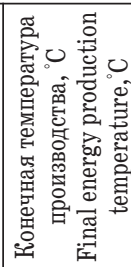 & 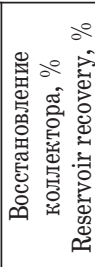 \\
\hline $10 \times 10$ & 100 & 625,7 & 85,4 & 92,5 & 69,0 \\
\hline $4 \times 25$ & 100 & 612,4 & 83,3 & 94,4 & 89,4 \\
\hline $2 \times 50$ & 100 & 588,3 & 82,4 & 95,4 & 97,2 \\
\hline
\end{tabular}

10-летний цикл эксплуатации-остановки позволяет выработать больше тепла, так как температура получаемой воды остается на более высоком уровне. В то же время самое высокое восстановление температуры происходит при наиболее продолжительном цикле эксплуатации-остановки. Это объясняется тем, что с увеличением продолжительности добычи возрастает разность температур в резервуаре, что приводит к более сильному температурному компенсационному притоку тепла [29] (рис. 9).

\section{Заключение}

Геотермальная энергия обладает огромным потенциалом для будущего обеспечения электричеством и теплом, имеет высокую доступность и распространненность. Энергия термальных вод является возобновляемой с низким (вплоть до нулевых значений) выбросом загрязняющих веществ при использовании. Одним из ключевых моментов разработки является вопрос устойчивости, при решении которого важен мониторинт и рациональный режим эксплуатации.

Исходя из результатов моделирования, снижение температуры воды продуктивной скважины Ханкальского месторождения начнется через 6-7 лет с начала разработки и является лишь вопросом времени. В то же время достижение устойчивости разработки и «неисчерпаемости» ресурса возможно с применением адекватной стратегии: «...для каждой геотермальной системы и для каждого способа эксплуатации существует определенный уровень максимального производства энергии, ниже которого можно будет поддерживать постоянное производство энергии из системы в течение очень долгого времени (100-300 лет)...» [3].

\section{СПИСОК ЛИТЕРАТУРЫ}

1. Термальные подземные воды Восточно-Предкавказского артезианского бассейна: экономические аспекты использования на примере Ханкальского месторождения / А.М. Фархутдинов, И.Ш. Хамитов, С.В. Черкасов, М.Ш. Минцаев, Ш.Ш. Заурбеков, А.А. Шаипов, М.М. Лабазанов // Известия Томского по-
Одним из основных преимуществ Ханкальского месторождения является наличие многопластовой системы. Вертикальное расширение «холодного фронта» ограничено непроницаемыми глинистыми прослоями, поэтому обратная закачка в один из продуктивных пластов не снижает температуру в других. В случае значительного снижения температуры в ходе разработки XIII пласта существует возможность пробурить новый «дублет» на той же территории на ресурс наиболее перспективных пластов IV-VII, XVI или XXII, что позволит геотермальной станции продолжить работу. Pесурс XIII пласта можно использовать снова после периода остановки с учетом относительно высокой скорости восстановления температуры. В перспективе возможно периодическое использование различных пластов.

Следует подчеркнуть важность размещения скважин параллельно двум главным разломам, при этом продуктивную скважину - в южной, нагнетательную - в северной части, поскольку естественное движение подземных вод способно замедлить расширение холодного фронта до продуктивной скважины.

Установка и периодическое использование двух или более циркуляционных систем могут быть наиболее оптимальным решением проблемы устойчивости при долгосрочной эксплуатации. Однако рациональность этого метода, требующего больших дополнительных затрат на начальном этапе его реализации (бурение составляет от 30 до $50 \%$ стоимости геотермального проекта [30]), представляет собой довольно сложный вопрос. Помимо гидрогеологических и геотермальных аспектов, следует учитывать экономические и технические особенности различных вариантов эксплуатации месторождений, то есть необходимо сотрудничество гидрогеологов, специалистов геотермии, технологов и экономистов.

Результаты моделирования показывают, что долгосрочное использование геотермальных вод на месторождении возможно, но адекватный сценарий эксплуатации требует всестороннего анализа этой проблемы. Адаптированные методы математического моделирования в дальнейшем могут быть использованы при обосновании условий эксплуатации других месторождений, а освоение экологически чистых геотермальных вод будет значительным вкладом в производство энергии и может изменить традиционные схемы энергоснабжения в ряде регионов России.

Исследование выполнено при финансовой поддержке РФФИ в рамках научного проекта № 18-35-00357.

литехнического университета. Инжиниринг георесурсов. 2017. - T. 328. - № 1. - C. 50-61.

2. The state and prospects for the utilization of geothermal resources in the Russian Federation / S.V. Cherkasov, T.G. Churikova, L.R. Bekmurzaeva, B.N. Gordeichik, A.M. Farkhutdinov // Ecology, Environment and Conservation. - 2015. - V. 21. - Suppl. Issue. - P. 67-77. 
3. Sustainable production of geothermal energy: suggested definition / G. Axelsson, A. Gudmundsson, B. Steingrimsson, G. Palmason, H. Armannsson, H. Tulinius, O.G. Flovenz, S. Bjornsson, V. Stefansson // IGA-News. - 2001. - № 43. - P. 1-2.

4. Cherkasov S.V., Farkhutdinov A.M., Rykovanov D.P., Shaipov A.A. The Use of Unmanned Aerial Vehicle for Geothermal Exploitation Monitoring: Khankala Field Example // Journal of Sustainable Development of Energy, Water and Environment Systems. - 2018. - № 6 (2). - P. 351-362.

5. Antics M., Papachristou M., Ungemach P. Sustainable heat mining. A reservoir engineering approach // Proc. of the $30^{\text {th }}$ Workshop on Geothermal Reservoir Engineering. - Stanford, California: Stanford University, January 31 - February 2, 2005. - 14 p.

6. Ungemach P., Antics M., Papachristou M. Sustainable Geothermal Reservoir Management // World Geothermal Congress. Turkey, 24-29 April 2005. - 12 p.

7. Ungemach P., Papachristou M., Antics M. Renewability versus Sustainability. A Reservoir Management Approach // European Geothermal Conference. - Unterhaching, Germany, 30 May 1 June 2007. - $13 \mathrm{p}$.

8. Ungemach P., Antics M., Lalos P. Sustainable geothermal reser voir management - a modelling suite // Proc. of Australian Geothermal Energy Conference. - Melbourne, Geoscience Australia, 16-18 November 2011. - P. 267-275.

9. Shortall R., Davidsdottir B., Axelsson G. Geothermal energy for sustainable development: A review of sustainability impacts and assessment frameworks / Renewable and Sustainable Energy Reviews. - 2015. - № 44. - P. 391-406.

10. Axelsson G. Sustainable management of geothermal resources // SDG Short Course I on Sustainability and Environmental Management of Geothermal Resource Utilization and the Role of Geothermal in Combating Climate Change, UNU-GTP and LaGeo. Santa Tecla, El Salvador, 2016. - 15 p.

11. Integrated research as key to the development of a sustainable geothermal energy technology / C. Meller, J. Bremer, S. Baur, T. Bergfeldt, P. Blum, T. Canic et al. // Energy Technology. 2017. - № 5 (7). - P. 965-1006.

12. Geothermal energy in deep aquifers: A global assessment of the resource base for direct heat utilization / J. Limberger, T. Boxemb, M. Pluymaekers, D. Bruhn, A. Manzella, P. Calcagno, F. Beekman, S. Cloetingh, J.-D. van Wees // Renewable and Sustainable Energy Reviews. - 2018. - № 82. - P. 961-975.

13. Всеволожский В.А. Основы гидрогеологии. 2-е. изд. - М.: Изд-во МГУ, 2007. - 448 с.

14. Diersch H.-J.G. FEFLOW - Finite element modeling of flow, mass and heat transport in porous and fractured media. - Springer, Berlin Heidelberg, 2014. - 996 p. DOI: 10.1007/978-3-642-38739-5.

15. McDonald M.G., Harbaugh A.W. A modular three-dimensional finite-difference ground-water flow model. Open-File Report 83-875. - U.S. Geological Survey, 1983.

16. Goblet P. Modélisation des transferts de masse et d'énergie en aquifère: PhD Thesis. - Paris, France, 1980. -200 p.
17. Pruess K. Mathematical modelling of fluid flow and heat transfer in geothermal systems. An introduction in five lectures. Berkeley National Laboratory Report LBNL-51295. -Berkeley, CA, 2002. $-84 \mathrm{p}$.

18. Thiéry D. Software MARTHE. Modelling of Aquifers with a Rectangular Grid 158 in Transient state for Hydrodynamic calculations of heads and flows. Release 4.3. Report $4 \mathrm{~S} / \mathrm{EAU}^{\circ}{ }^{\circ}$ R32548. - BRGM, 1990.

19. OpenGeoSys: an open-source initiative for numerical simulation of thermo-hydro-mechanical/chemical (THM/C) processes in porous media / 0. Kolditz, S. Bauer, L. Bilke, N. Böttcher, J.0. Delfs, T. Fischer et al. // Environmental Earth Sciences. 2012. - № 67 (2). - P. 589-599.

20. Курбанов М.К. Геотермальные и гидроминеральные ресурсы Восточного Кавказа и Предкавказья. - М.: Наука, 2001. - 260 с.

21. Дежникова И.Ю. Карта запасов подземных вод и степени их освоения по гидрогеологическим структурам территории Российской Федерации. - 2015. URL: http://www.geomonitoring.ru/ (дата обращения: 26.12.2018).

22. Алхасов А.Б. Возобновляемые источники энергии. - М.: МЭИ, 2011. $-270 \mathrm{c}$.

23. Farkhutdinov A., Goblet P., Fouquet de C., Cherkasov S. A case study of the modeling of a hydrothermal reservoir: Khankala deposit of geothermal waters // Geothermics. - 2016. - V. 59. P. 56-66. URL: http://dx.doi.org/10.1016/j.geothermics.2015.10.005. (дата обращения: 26.12.2018).

24. Farkhutdinov A.M., Goblet P., Cherkasov S.V. Computer modelling in geothermal waters reservoirs exploitation on the example of the Khankala deposit // Ecology, Environment and Conservation. - 2015. - V. 21. -Suppl. Issue. - P. 87-91.

25. The use of computer modelling to forecast the sustainability in the development of geothermal waters resource: Khankala deposit example / A. Farkhutdinov, P. Goblet, C. de Fouquet, R. Ismagilov, I. Farkhutdinov, S. Cherkasov // International Journal of Renewable Energy Research. - 2015. - V. 5. - № 4. - P. 1062-1068.

26. Marsily G. Hydrogéologie quantitative. - Paris: Masson, 1981. $215 \mathrm{p}$.

27. Gringarten A.C., Sauty J.P. A theoretical study of heat extraction from aquifers with uniform regional flow // Journal of Geophysical Research. - 1975. - № 35. - P. 4956-4962.

28. Ресурсы термальных вод СССР / под ред. С.С. Бондаренко. М.: Наука, 1975. -240 c.

29. Megel T., Rybach L. Production capacity and sustainability of geothermal doublets // Proc. World Geothermal Congress. - Kyushu-Tohoku, Japan, May 28 - June 10, 2000. - P. 859-854.

30. Dumas P., Antics M., Ungemach P. GeoELEC - Report on Geothermal Drilling / Geoelec. - 2011. - 36 p. URL: http://www.geoelec.eu/wp-content/uploads/2011/09/D-3.3GEOELEC-report-on-drilling.pdf (дата обращения: 26.12.2018).

Поступила 15.01.2019 2.

\section{Информация об авторах}

Фархутдинов A.M., кандидат геолого-минералогических наук, доцент кафедры геологии и полезных ископаемых географического факультета Башкирского государственного университета.

Фархутдинов И.М., кандидат геолого-минералогических наук, заведующий кафедрой геологии и полезных ископаемых географического факультета Башкирского государственного университета.

Черкасов С.B., кандидат геолого-минералогических наук, директор Государственного геологического музея им. В.И. Вернадского.

Исмагилов P.A., кандидат геолого-минералогических наук, старший научный сотрудник Института геологии УФИЦ РАН; доцент кафедры геофизики Башкирского государственного университета.

Хайрулина Л.А., старший преподаватель кафедры геологии и полезных ископаемых географического факультета Башкирского государственного университета. 
UDC 553.78

\title{
USE OF COMPUTER MODELLING FOR THE KHANKALA GEOTHERMAL WATERS SUSTAINABLE EXPLOITATION
}

\author{
Anvar M. Farkhutdinov', \\ anvarfarh@mail.ru \\ Iskhak M. Farkhutdinov', \\ iskhakgeo@gmail.com \\ Sergey V. Cherkasov², \\ sergy@sgm.ru \\ Rustem A. Ismagilov 3.1, \\ rustem_ismagilov@bk.ru \\ Larisa A. Khairulina', \\ artthemix@mail.ru \\ 1 Bashkir State University, \\ 32, Zaki Validi street, Ufa, 450076, Russia. \\ 2 Vernadsky State Geological Museum, \\ 11/11, Mokhovaya street, Moscow, 125009, Russia. \\ ${ }^{3}$ Institute of Geology, Ufa Federal Research Center of Russian Academy of Sciences, \\ 16/2, Karl Marx street, Ufa, 450077, Russia
}

The relevance of the research is caused by the need of achieving sustainability in exploitation of geothermal waters. Temperature drop of geothermal reservoir during the re-injection of used fluid is the most important problem of long-term operation, the forecast of which is possible with the help of computer modelling.

The main aim of the research is to forecast the temperature changes during the Khankala field exploitation, consider various development scenarios and propose possible solutions to achieve sustainability in field operation.

Objects of the research are the Khankala field; doublet circulation system of the Khankala geothermal station.

Methods: computer modelling of temperature changes of the geothermal resource; assessment and analysis of possible variants for periodic exploitation of the field; calculation of the total heat generation and the final temperature of the geothermal reservoir at the time of shutdown for various operating options.

Results. Temperature drop in a production well will start after 6-7 years of exploitaion in the planned mode at the Khankala field. Periodic short-term operation and shutdown (10-year cycle, 100 years in total) allows more heat to be generated compared with longer operation (50-year cycle, 100 years in total), while the second variant makes it possible to achieve a higher temperature recovery in the production well. In the case of a significant decrease in temperature after a certain period of the XIII layer exploitation, periodic use of various productive layers (IV-VII, XVI or XXII) is proposed to ensure the sustainability of the geothermal station. Development of the XIII layer is possible after a period of stopping due to the relatively high rate of temperature recovery. Wells should be placed parallel to the two main faults of the Khankala field, with the production well in the south and the injection well in the northern part, since the natural flow of groundwater can slow down the expansion of the cold front to the production well. The installation and periodic use of two or more circulation systems can provide the greatest stability in long-term operation. However, the rationality of this method, which requires large additional costs at the initial stage of project implementation, is a complex issue. The choice of operating mode requires consideration of hydrogeological, geothermal, economic and technical aspects with the joint participation of hydrogeologists, geothermal specialists, technologists and economists.

Key words:

Renewable energy resources, geothermal waters, sustainable exploitation, doublet, modelling, Khankala field.

The research was financially supported by the RFBR within the scientific project no. 18-35-00357.

\section{REFERENCES}

1. Farkhutdinov A.M., Khamitov I.Sh., Cherkasov S.V., Mintsaev M.Sh., Zaurbekov Sh.Sh., Shaipov A.A., Labazanov M.M. Geothermal waters of East-Ciscausasian Artesian Basin: economical aspects of using by the example of Khankala deposit, Bulletin of the Tomsk Polytechnic University. Geo Assets Engineering, 2017, vol. 328, no 1, pp. 50-61.

2. Cherkasov S.V., Churikova T.G., Bekmurzaeva L.R., Gordeichik B.N., Farkhutdinov A.M. The state and prospects for the utilization of geothermal resources in the Russian Federation, Ecology, Environment and Conservation, 2015, vol. 21, Suppl. Issue, pp. 67-77.

3. Axelsson G., Gudmundsson A., Steingrimsson B., Palmason G., Armannsson H., Tulinius H., Flovenz 0.G., Bjornsson S., Stefansson V. Sustainable production of geothermal energy: suggested definition. IGA-News, 2001, no. 43, pp. 1-2.

4. Cherkasov S.V., Farkhutdinov A.M., Rykovanov D.P., Shaipov A.A. The Use of Unmanned Aerial Vehicle for Geothermal 
Exploitation Monitoring: Khankala Field Example, Journal of Sustainable Development of Energy, Water and Environment Systems, 2018, no. 6 (2), pp. 351-362.

5. Antics M., Papachristou M., Ungemach P. Sustainable heat mining. A reservoir engineering approach, Proc. of the $30^{\text {th }}$ Workshop on Geothermal Reservoir Engineering. Stanford, California, Stanford University, January 31 - February 2, 2005. $14 \mathrm{p}$.

6. Ungemach P., Antics M., Papachristou M. Sustainable Geothermal Reservoir Management. World Geothermal Congress. Turkey, 24-29 April 2005. 12 p.

7. Ungemach P., Papachristou M., Antics M. Renewability versus sustainability. A reservoir management approach. European Geothermal Conference. Unterhaching, Germany, 30 May 1 June 2007. $13 \mathrm{p}$.

8. Ungemach P., Antics M., Lalos P. Sustainable geothermal reservoir management - a modelling suite. Proc. of Australian Geother mal Energy Conference. Melbourne, Geoscience Australia, 16-18 November 2011. pp. 267-275.

9. Shortall R., Davidsdottir B., Axelsson G. Geothermal energy for sustainable development: a review of sustainability impacts and assessment frameworks. Renewable and Sustainable Energy Reviews, 2015, no. 44, pp. 391-406.

10. Axelsson G. Sustainable management of geothermal resources. SDG Short Course I on Sustainability and Environmental Management of Geothermal Resource Utilization and the Role of Geothermal in Combating Climate Change. UNU-GTP and LaGeo. Santa Tecla, El Salvador, 2016. 15 p.

11. Meller C., Bremer J., Baur S., Bergfeldt T., Blum P., Canic T. Integrated research as key to the development of a sustainable geothermal energy technology. Energy Technology, 2017, no. 5 (7), pp. 965-1006.

12. Limberger J., Boxemb T., Pluymaekers M., Bruhn D., Manzella A., Calcagno P., Beekman F., Cloetingh S., Van Wees J.-D. Geothermal energy in deep aquifers: a global assessment of the resource base for direct heat utilization. Renewable and Sustainable Energy Reviews, 2018, no. 82, pp. 961-975.

13. Vsevolozhsky V.A. Osnovy gidrogeologii [Fundamentals of hydrogeology]. Moscow, MSU Publ., 2007. 448 p.

14. Diersch H.-J.G. FEFLOW - Finite element modeling of flow, mass and heat transport in porous and fractured media. Berlin Heidelberg, Springer, 2014. 996 p. DOI: 10.1007/978-3-642-38739-5.

15. McDonald M.G., Harbaugh A.W. A modular three-dimensional finite-difference ground-water flow model. Open-File Report 83-875. U.S. Geological Survey, 1983.

16. Goblet P. Modélisation des transferts de masse et d'énergie en aquifère [Modelling mass and energy transfers in aquifers]. $\mathrm{PhD}$ Thesis. Paris, France, 1980. 200 p.

17. Pruess K. Mathematical modelling of fluid flow and heat transfer in geothermal systems. An introduction in five lectures. Berkeley National Laboratory Report LBNL-51295. Berkeley, CA, 2002. $84 \mathrm{p}$.
18. Thiéry D. Software MARTHE. Modelling of Aquifers with a Rectangular Grid 158 in Transient state for Hydrodynamic calculations of heads and flows. Release 4.3. Report 4S/EAU n R32548. BRGM, 1990.

19. Kolditz 0., Bauer S., Bilke L., Böttcher N., Delfs J.0., Fischer T. OpenGeoSys: an open-source initiative for numerical simulation of thermo-hydro-mechanical/chemical (THM/C) processes in porous media. Environmental Earth Sciences, 2012, no. 67 (2), pp. 589-599.

20. Kurbanov M.K. Geotermalnye $i$ gidromineralnye resursy Vostochnogo Kavkaza i Predkavkazya [Geothermal and hydromineral resources of the Eastern Caucasus and Ciscaucasia]. Moscow, Nauka Publ., 2001. 260 p.

21. Dezhnikova I.Yu. Karta zapasov podzemnykh vod $i$ stepeni ikh osvoeniya po gidrogeologicheskim strukturam territorii Rossiyskoy Federatsii [Map of groundwater reserves and degree of their development by hydrogeological structures of the territory of the Russian Federation]. 2015. Available at: http://www.geomonitoring.ru/ (accessed 26 December 2018).

22. Alkhasov A.B. Vozobnoulyaemye istochniki energii [Renewable energy sources]. Moscow, MEI Publ., 2011. 270 p.

23. Farkhutdinov A., Goblet P., Fouquet de C., Cherkasov S. A case study of the modeling of a hydrothermal reservoir: Khankala deposit of geothermal waters. Geothermics, 2016, vol. 59, pp. 56-66. Available at: http://dx.doi.org/10.1016/j.geothermics.2015.10.005 (accessed 26 December 2018).

24. Farkhutdinov A.M., Goblet P., Cherkasov S.V. Computer modelling in geothermal waters reservoirs exploitation on the example of the Khankala deposit. Ecology, Environment and Conservation, 2015, vol. 21, Suppl. Issue, pp. 87-91.

25. Farkhutdinov A., Goblet P., de Fouquet C., Ismagilov R., Farkhutdinov I., Cherkasov S. The use of computer modelling to forecast the sustainability in the development of geothermal waters resource: Khankala Deposit Example. International Journal of Renewable Energy Research, 2015, vol. 5, no. 4, pp. 1062-1068.

26. Marsily G. Hydrogéologie quantitative [Quantitative hydrogeology]. Paris, Masson, 1981. 215 p.

27. Gringarten A.C., Sauty J.P. A theoretical study of heat extraction from aquifers with uniform regional flow. Journal of $\mathrm{Ge}$ ophysical Research, 1975 , no. 35, pp. 4956-4962.

28. Resursy termalnykh vod SSSR [Resources of geothermal waters of the USSR]. Ed. by S.S. Bondarenko. Moscow, Nauka Publ., $1975.240 \mathrm{p}$.

29. Megel T., Rybach L. Production capacity and sustainability of geothermal doublets. Proc. World Geothermal Congress. KyushuTohoku, Japan, May 28 - June 10, 2000. pp. 859-854.

30. Dumas P., Antics M., Ungemach P. GeoELEC - Report on Geothermal Drilling. Geoelec. 2011, 36 p. Available at: http://www.geoelec.eu/wp-content/uploads/2011/09/D-3.3GEOELEC-report-on-drilling.pdf (accessed 26 December 2018).

Received: 15 January 2019.

\section{Information about the authors}

Anvar M. Farkhutdinov, Cand. Sc., associate professor, Bashkir State University.

Iskhak M. Farkhutdinov, Cand. Sc., associate professor, Bashkir State University.

Sergey V. Cherkasov, Cand. Sc., director, Vernadsky State Geological Museum.

Rustem A. Ismagilov, Cand. Sc., senior researcher, Institute of Geology, Ufa Federal Research Center of Russian Academy of Sciences; associate professor, Bashkir State University.

Larisa A. Khairulina, assistant professor, Bashkir State University. 